\title{
Personalized treatment: the future of medicine. a perspective on the preconditioning for stem cell transplantation
}

\section{Introduction}

Each individual is unique and, accordingly, so should the medical care. Medical treatment needs to be individually tailored for optimal efficiency and minimal side effects, which can be described as personalized medicine. Although the term 'personalized medicine' can be tracked backed to 1971, it was not until 2000 that it was given serious consideration. Since then, this field has an annual increase in publications of $49 \% .^{1}$ Different authors provide diverse perceptions of personalized therapy. While some authors concentrate on mapping the functions of various genetic polymorphisms, others study the metabolic activity. Some scientists describe personalized medicine as monitoring of drug levels in the blood and adjusting the dose accordingly, while others investigate the mechanisms underlying the side effects. Even the target of personalized medicine varies from medically focused, such as achieving the best results with least side effects, to economically directed, such as saving the funding for unnecessary or ineffective therapeutics and avoiding hospitalization days as well as preventing long term morbidity and loss of productivity. ${ }^{2}$ For this reason, Schleidgen et al. ${ }^{1}$ analyzed 2457 articles published in the field of personalized medicine in order to report a solid definition for such a title. Their suggestion was "Personalized medicine seeks to improve stratification and timing of health care by utilizing biological information and biomarkers on the level of molecular disease pathways, genetics, proteomics as well as metabolomics". ${ }^{1}$ The Food and Drug Administration (FDA) provided an easier explanation in its report 'Paving the Way for Personalized Medicine' in 2013, which was "providing the right patient with the right drug at the right dose at the right time". In spite of the importance of personalized medicine in diagnostics and other modalities of management, we will concentrate here on the personalization of medical treatment, taking our experience in the conditioning process prior to hematopoietic stem cell therapy (SCT) as an example.

\section{Pharmacogenomics}

The secret of our uniqueness is hidden in our DNA and similarly is our response to drugs. Pharmacogenomics is a branch of science that correlates the metabolism of drugs to the genetic variation which is reflected in the activity or the isoform of the involved enzymes. The most obvious example is the different members of cytochrome P450 (reviewed in). ${ }^{3}$ Pharmacogenomics can be the considered the compass for personalized medicine, or as Ratain ${ }^{4}$ described in 2007, its GPS. The author described three components for such a navigation system; the first is the knowledge of the individual's genome sequence, the second is accessibility to such information and the third is to understand how to interpret it. ${ }^{4}$ After the completion of the human genome project, the breakthrough occurred. At least 121 drugs are currently FDAapproved for patients with a certain Pharmacogenomics biomarkers or polymorphisms. ${ }^{3,5,6}$

In 2006, Senator Barack Obama looked at Pharmacogenomics
Volume 2 Issue 3 - 2015

\author{
Moustapha Hassan,' Ahmed El-Serafi ${ }^{2}$ \\ 'Department of Laboratory Medicine, Karolinska Institute, \\ Sweden \\ ${ }^{2}$ Department of Basic Medical Sciences, University of Sharjah, \\ United Arab Emirates
}

Correspondence: Moustapha Hassan, Department of Laboratory Medicine, Karolinska Institute, Sweden, Tel +46858583862, Email moustapha.hassan@ki.se

Received: August 27, 2015 | Published: September 0I, 2015

from a financial angle and introduced to the US Senate a project to secure funding for the development of this new branch of science. He suggested the formation of a national Biobank for this purpose, taking Sweden as a model in this context. Unfortunately, Congress suggested a foundation rather than federal involvement. ${ }^{4}$ Taking all this into consideration, the $\$ 1000$ genome project was born. This project aims to develop a technology to provide an individual's entire genome sequence at a cost of $\$ 1000$, which is being approached ${ }^{7}$ While the genomic constituent of the patient will affect the response to treatment in terms of metabolism, the acquired mutations, e.g. in a tumor, will determine the resistance to such treatment. Currently, there is a growing body of hospital-based genotyping centers in the United States that provide help in shaping 'personalized' management plans. Biopsies of the tumor are assayed for key mutations and therapy is planned accordingly. ${ }^{6}$ The future direction is to detect newly acquired mutations in a tumor from circulating free DNA; i.e. by collecting a peripheral blood sample. ${ }^{8}$

\section{Hematopoietic stem cell therapy}

Hematopoietic stem cell therapy is a curative treatment for patients with hematological malignancies such as leukemia, my elodysplastic syndrome and myeloma as well as several inherited metabolic disorders. These cells are separated from the bone marrow by immuno selection of CD34+ antigen but can also be isolated from other sources, such as peripheral blood or umbilical cord blood. Upon injection, the cells will home in on the bone marrow to replenish their numbers and differentiate into mature blood cells. For this purpose, the bone marrow needs to be cleared from the original cells, which are in most cases malignant cells, in order to give the injected cells space and to avoid having an immune rejection. ${ }^{9}$ The process of wiping the original cells is called conditioning for SCT. Conditioning regimen is a crucial component of SCT that facilitates the engraftment of donor stem cells and contributes to a curative treatment by reducing the tumor burden. The conditioning regimen is selected with regard to different factors such as the diagnosis (Figure 1), age of the patient, co-morbidity, type of donor and HLA-matching, taking into consideration the risk for transplantation-related complications. ${ }^{10,11}$ 


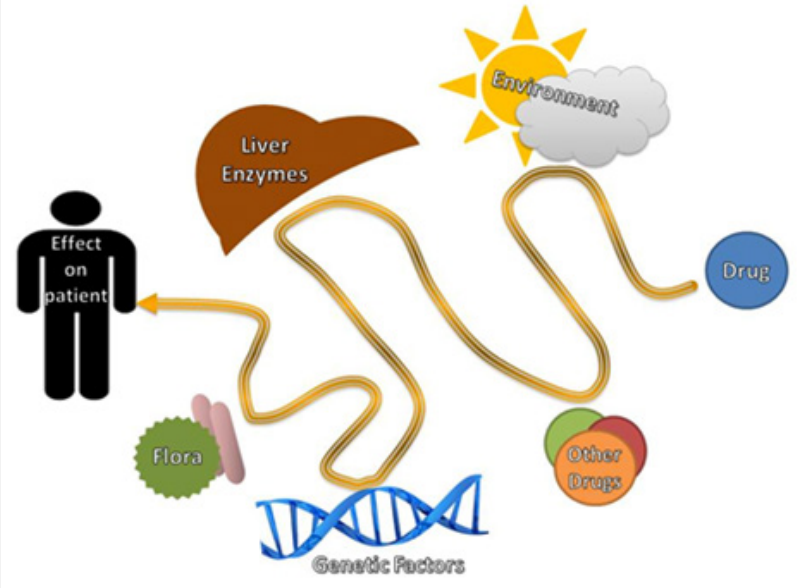

Figure I The effect of the drug on the patient is determined by many factors including genetic make-up, liver enzyme activity, gut flora, and effect of the environment as well as interaction with other drugs.

Although SCT is currently standard procedure, too many patients still experience life-threatening complications such as interstitial pneumonia, hyper pigmentation, seizures, sinusoidal obstruction syndrome (SOS), emesis, juvenile arthritis and autoimmune disease. Urotoxicity, gut toxicity, cardiotoxicity and graft versus host disease are also common complications during SCT. ${ }^{12,13}$ For several years, our team has investigated various aspects of the conditioning process that influence the efficacy of the treatment, the frequency and severity of side effects as well as the possible complications and their management. ${ }^{14-16}$

Despite advances in molecular and cellular biology and development of new targeted drugs, the conventional cytostatics remain the corner stone of preconditioning for SCT as well as for management of many types of cancer. Alkylating agents are preferred because of their effect on non-dividing malignant cells. ${ }^{11}$ The bioavailability of these drugs is correlated with several factors including age of the patient, genetic make-up, gut function, gut flora, and types of ingested food, in addition to the chemical and physical properties of the drug. ${ }^{17,18}$ In a multicentric phase III clinical trial, low doses of cytostatics were compared to higher doses with regard to the outcome of SCT and survival. The study was permanently halted as higher doses were clearly associated with a better prognosis. ${ }^{11,19}$

\section{Cyclophosphamide}

One of these cytostatics is Cyclophosphamide (Cy), a nitrogen mustard alkylating agent that can be used alone or in combination with total body irradiation and/or other drugs. After being activated in the liver, Cy causes cross linking of DNA which inhibits the cell replication and favors its apoptosis. In an interesting study on breast cancer patients, four polymorphisms involved in Cy metabolism were investigated. There were no alleles associated with disease-free survival, but a certain polymorphism in the glutathione-S-transferase 1 was protective against hematological side effects. ${ }^{20}$ Recently, we reported for the first time - the role of the cytochrome CYP2J2 in Cybio-activation to 4-Hydroxy-Cy (4-OH-Cy) in patients, cells and microsomes. CYP2J2 expression was significantly up regulated in patients with hematological malignancies $(\mathrm{P}<0.01)$ and in patients undergoing SCT and conditioned with high dose $\mathrm{Cy}(\mathrm{P}<0.05)$ in comparison to healthy controls. The concentration ratio of the active metabolite 4-OH-Cy/Cy (as an index for bioactivation) showed significant $(\mathrm{P}<0.05)$ correlation with $\mathrm{CYP} 2 \mathrm{~J} 2$ expression. Repeated
$\mathrm{Cy}$ administration showed increased 4-OH-Cy/Cy ratio, indicating auto-induction of the relevant cytochromes. In vitro, the survival of HL 60 cells was improved when incubated with Cy combined with the CYP2J2 inhibitor, telmisartan. In this case, the production of 4-OHCy was reduced by $50 \%$ in comparison with $\mathrm{Cy}$ treatment alone. ${ }^{21}$

\section{Busulphan}

Busulphan $(\mathrm{Bu})$ is another cytostatic drug that is widely used in SCT conditioning, either alone or more commonly in combination with $\mathrm{Cy}$. Bu is an alkylating agent that attacks the DNA double strands, forming cross linking and leading to apoptosis. The therapeutic window for Bu is very narrow. Our team is involved in the therapeutic drug monitoring of $\mathrm{Bu}$ in different hospitals in Sweden as well as other European countries. The main goal is to personalize Bu therapy in order to reach the maximum treatment efficacy with the lowest side effects. We developed a 'limited sampling model' that involves fewer number of blood samples in order to estimate the area under the plasma concentration time curve of $\mathrm{Bu}$ and adjust the following dose accordingly. ${ }^{22}$ This model is particularly of great importance to pediatric or anemic patients as well as for the nursing staff, and also when the cost of treatment should be considered. Thus more patients can currently obtain a second chance to catch outliers in absorption or metabolism, which makes up for the lost precision.

Route of administration plays an important role in determining the efficacy of any drug, including $\mathrm{Bu}$. This drug is poorly dissolved in water and hence $\mathrm{Bu}$ is administrated orally as tablets or intravenously dissolved in dimethylacetamide. Both forms of the drug are associated with variation in the bioavailability up to 6 fold in children and 2 fold in adults. ${ }^{15}$ Although intravenous $\mathrm{Bu}$ was expected to have superior properties, we have reported undesirable drug kinetics (in press) and the continuous need for dose adjustment. In a previous study on 34 patients, intravenous Bu needed to be adjusted - at least once - in $68 \%$ of the patients. The dose increased between $7-33 \%$ in $70 \%$ of those patients, while in the rest $(30 \%)$ the dose had to be decreased by $7-20 \% .{ }^{23}$ The variability in systemic $\mathrm{Bu}$ exposure is caused by differences in the individual hepatic metabolism, genetic polymorphisms in glutathione-S-transferase, age, initial diagnosis and concomitant medication. Such variability is more obvious in children.

The development of alternative methods for the administration of $\mathrm{Bu}$ is still a target to achieve efficacy and safety. In the late 1990s, we developed a liposomal formulation of $\mathrm{Bu}$, which could be infused intravenously over the course of three hours. It is associated with better pharmacokinetic properties and needs less strict monitoring. The dose is calculated based on the body surface area. This formulation has passed phase II in clinical trials. ${ }^{24}$ Recently, we reported new nanocarriers of polylactic -co-glycolic acid that encapsulated $\mathrm{Bu}$ and the particles were followed up by magnetic resonance imaging in vivo and showed localization in the liver, lungs and to a lesser extent in the spleen in an experimental model. In vitro, the nanocarrier degradation and drug release were recorded. This new carrier can be another new development in $\mathrm{Bu}$ delivery. ${ }^{25}$

Today, the combination of $\mathrm{Cy}$ and $\mathrm{Bu}$ is very common in preconditioning and has a similar effect to $\mathrm{Cy}$ plus total body irradiation. ${ }^{11}$ The administration of these drugs has to be carefully planned as the time interval between the last dose of $\mathrm{Bu}$ and the first dose of Cy would influence the pharmacokinetics and metabolism of $\mathrm{Cy}$. We have shown earlier that if $\mathrm{Cy}$ was administrated in less than $24 \mathrm{~h}$ after the last dose of $\mathrm{Bu}$, the treatment efficacy was significantly lower and SOS and mucositis were evident as side effects. ${ }^{26}$ 
In the future, we hope that a simple blood test could tell doctors the genotype and polymorphisms of the patient, and the treatment individualized accordingly. Various forms of administration, therapeutic drug monitoring and targeted drug therapy could minimize - or in some cases eliminate - the side effects and maximize efficacy. This future perspective is the target of medical research today.

\section{Conclusion}

Personalized treatment is a modern approach of management which depends on tailoring the drug regimen according to the patient's genetic background which, in turn, affects the metabolism of the drug. The accumulation of a drug in the body causes side effects, while a low drug exposure will decrease the efficacy; both are associated with financial loss. Identifying the enzymes responsible for each drug metabolism and understanding how different polymorphisms will affect them is a key component of personalized medicine. The conditioning of SCT is a living example that could be used as a model for other management plans.

\section{Acknowledgements}

None.

\section{Conflict of interest}

The author declares no conflict of interest.

\section{References}

1. Schleidgen $\mathrm{S}$, Klingler C, Bertram T, et al. What is personalized medicine: sharpening a vague term based on a systematic literature review. BMC Med Ethics. 2013;14:55.

2. Abtin A, Jain R, Mitchell AJ, et al. Perivascular macrophages mediate neutrophil recruitment during bacterial skin infection. Nat Immunol. 2014;15(1):45-53

3. Schwab M, Schaeffeler E. Pharmacogenomics: a key component of personalized therapy. Genome Med. 2012;4(11):93.

4. Ratain MJ. Personalized medicine: building the GPS to take us there Clin Pharmacol Ther. 2007;81(3):321-322.

5. Shu WY, Li JL, Wang XD, et al. Pharmacogenomics and personalized medicine: a review focused on their application in the Chinese population. Acta Pharmacol Sin. 2015;36(5):535-543.

6. Chabner BA, Ellisen LW, Iafrate AJ. Personalized medicine: hype or reality. Oncologist. 2013;18(6):640-643.

7. Hayden EC. Technology: The $\$ 1,000$ genome. Nature. 2014;507(7492):294-295.

8. Lebofsky R, Decraene C, Bernard V, et al. Circulating tumor DNA as a non-invasive substitute to metastasis biopsy for tumor genotyping and personalized medicine in a prospective trial across all tumor types. $\mathrm{Mol}$ Oncol. 2015;9(4):783-790.

9. Suarez-Alvarez B, Lopez-VazquezA, Lopez-Larrea C. Mobilization and homing of hematopoietic stem cells. Adv Exp Med Biol. 2012;741:152170 .

10. Hassan M, Andersson BS. Role of pharmacogenetics in busulfan/ cyclophosphamide conditioning therapy prior to hematopoietic stem cell transplantation. Pharmacogenomics. 2013;14(1):75-87.
11. Gyurkocza B, Sandmaier BM. Conditioning regimens for hematopoietic cell transplantation: one size does not fit all. Blood. 2014;124(3):344 353.

12. Daikeler T, Tichelli A, Passweg J. Complications of autologous hematopoietic stem cell transplantation for patients with autoimmune diseases. Pediatr Res. 2012;71(4 Pt 2):439-444.

13. Haverman TM, Raber-Durlacher JE, Rademacher WM, et al. Oral complications in hematopoietic stem cell recipients: the role of inflammation. Mediators Inflamm. 2014;2014:378281.

14. Le Blanc K, Rasmusson I, Sundberg B, et al. Treatment of severe acute graft-versus-host disease with third party haploidentical mesenchymal stem cells. Lancet. 2004;363(9419):1439-1441.

15. Hassan M, Ljungman P, Bolme P, et al. Busulfan bioavailability. Blood. 1994;84(7):2144-2150

16. Nilsson $\mathrm{C}$, Aschan J, Hentschke P, et al. The effect of metronidazole on busulfan pharmacokinetics in patients undergoing hematopoietic stem cell transplantation. Bone Marrow Transplant. 2003;31(6):429-435.

17. Wilkinson GR. The effects of diet, aging and disease-states on presystemic elimination and oral drug bioavailability in humans. $A d v$ Drug Deliv Rev. 1997;27(2-3):129-159.

18. Al Hashmi S, Sadeghi B, Hassan Z, et al. Omega-3 from fish oil augments GVHD through the enhancement of chemotherapy conditioning regimen and selective FoxP3 depletion. Bone Marrow Transplantation. 2013;48(6):843-848.

19. Walter RB, Gyurkocza B, Storer BE, et al. Comparison of minimal residual disease as outcome predictor for AML patients in first complete remission undergoing myeloablative or nonmyeloablative allogeneic hematopoietic cell transplantation. Leukemia. 2015;29(1):137-144.

20. Yao S, Barlow WE, Albain KS, et al. Gene polymorphisms in cyclophosphamide metabolism pathway, treatment-related toxicity, and disease-free survival in SWOG 8897 clinical trial for breast cancer. Clin Cancer Res. 2010;16(24):6169-6176.

21. El-Serafi I, Fares M, Abedi-Valugerdi M, et al. Cytochrome P450 2J2, a new key enzyme in cyclophosphamide bioactivation and a potential biomarker for hematological malignancies. Pharmacogenomics J. 2015;15(5):405-413

22. Sjoo F, El-Serafi I, Enestig J, et al. Comparison of algorithms for oral busulphan area under the concentration-time curve limited sampling estimate. Clin Drug Investig. 2014;34(1):43-52.

23. Malar R, Sjoo F, Rentsch K, et al. Therapeutic drug monitoring is essential for intravenous busulfan therapy in pediatric hematopoietic stem cell recipients. Pediatr Transplant. 2011;15(6):580-588.

24. Hassan M, Nilsson C, Hassan Z, et al. A phase II trial of liposomal busulphan as an intravenous myeloablative agent prior to stem cell transplantation: $500 \mathrm{mg} / \mathrm{m}^{2}$ as a optimal total dose for conditioning. Bone Marrow Transplant. 2002;30(12):833-841.

25. Ye F, Barrefelt A, Asem $\mathrm{H}$, et al. Biodegradable polymeric vesicles containing magnetic nanoparticles, quantum dots and anticancer drugs for drug delivery and imaging. Biomaterials. 2014;35(12):3885-3894.

26. Hassan M, Ljungman $\mathrm{P}$, Ringden $\mathrm{O}$, et al. The effect of busulphan on the pharmacokinetics of cyclophosphamide and its 4-hydroxy metabolite: time interval influence on therapeutic efficacy and therapy-related toxicity. Bone Marrow Transplant. 2000;25(9):915-924. 